\title{
Reverse genetics tools in zebrafish: a forward dive into endocrinology
}

\author{
Sugano, Yuya ; Neuhauss, Stephan C F
}

\begin{abstract}
The zebrafish is a powerful genetic model organism. In recent years, zebrafish has been increasingly used to model human diseases. Due to a number of recent technological advancements, the genetic tool box is now also stocked with sophisticated transgenic and reverse genetic tools. Here, we focus on both commonly used and recently established reverse genetic and transgenic tools available in zebrafish. These new developments make the zebrafish an even more attractive animal model in comparative endocrinology.
\end{abstract}

DOI: https://doi.org/10.1016/j.ygcen.2013.02.002

Posted at the Zurich Open Repository and Archive, University of Zurich

ZORA URL: https://doi.org/10.5167/uzh-80718

Journal Article

Accepted Version

Originally published at:

Sugano, Yuya; Neuhauss, Stephan C F (2013). Reverse genetics tools in zebrafish: a forward dive into endocrinology. General and Comparative Endocrinology, 188:303-308.

DOI: https://doi.org/10.1016/j.ygcen.2013.02.002 


\section{Reverse Genetics Tools in Zebrafish: A Forward Dive into Endocrinology}

Yuya Sugano $^{1,2}$ and Stephan C.F. Neuhauss ${ }^{1}$

University of Zurich, ${ }^{1}$ Institute of Molecular Life Sciences, Institute of Anatomy ${ }^{2}$,

Neuroscience Center Zurich ${ }^{1}$ and Center for Integrative Human Physiology ${ }^{1,2}$,

Winterthurerstrasse 190, CH-8057 Zurich, Switzerland

Contact:

Stephan C.F. Neuhauss

Institute of Molecular Life Sciences, University of Zurich, Winterthurerstrasse 190, CH-8057

Zurich, Switzerland. Email: stephan.neuhauss@imls.uzh.ch

Phone: +41446356040

\section{Abbreviations:}

TALEN, transcription activator-like effector nucleases

ZFN, zinc finger nucleases

TILLING, targeted induced local lesions in genomes

UAS, upstream activating sequence 


\begin{abstract}
The zebrafish is a powerful genetic model organism. In recent years, zebrafish (Danio rerio) has been increasingly used to model human diseases. Due to a number of recent technological advancements, the genetic tool box is now also stocked with sophisticated transgenic and reverse genetic tools. Here, we focus on both commonly used and recently established reverse genetic and transgenic tools available in zebrafish. These new developments make the zebrafish an even more attractive animal model in comparative endocrinology.
\end{abstract}




\section{Introduction}

The last three decades have seen the establishment of the zebrafish as a major vertebrate model organism for studies of human diseases. The zebrafish made its first splash as a model for genetic control of vertebrate development due to its favorable biological characteristics such as high fecundity, transparency and external development of embryos [45]. Zebrafish genetics entered the picture and gained prominence with the two first large-scale mutagenesis screens performed in any vertebrate species $[8,16]$. At this time in the 1990 s and in the following years, zebrafish genetics was nearly exclusively confined to forward genetic studies, exploiting the huge number of available mutant strains, many of which proofed to be relevant for our understanding of mechanisms of pathogenesis of a number of human diseases $[12,28]$. Genetic studies of human disorders benefit from the investigation of specific target genes of interest. In contrast to forward genetics, the lack of tools to manipulate specific genes of interest hindered the realization of the full potential of the zebrafish for modeling human diseases [43]. However, recent technological advances, mainly in anti-sense morpholino mediated gene knockdown, transgenics using the Tol2 system and the induction of heritable genetic alterations with zinc finger nucleases, TALENs and TILLING technology heralds a new era in zebrafish reverse genetics.

The strengths of zebrafish have been largely realized in the study of developmental biology, as is also the case for endocrine systems in zebrafish [29]. Studies on zebrafish endocrine systems have revealed that the endocrine systems between humans and zebrafish are remarkably well conserved [30]. Significantly, studies on zebrafish endocrine systems show that the development of endocrine systems mostly complete within 5 days post fertilization (dpf) in zebrafish. In other words, it is possible to model the human endocrine systems in developing zebrafish, taking full advantage of the strengths of this animal model. 
In this review, we discuss several reverse genetics approaches that have been established in zebrafish as well as emerging techniques for targeted gene knockout including TALENs, and their application for studies of human endocrine systems.

\section{Reverse Genetic Approaches in Zebrafish}

The mouse model has been a dominant system to study disease related genes because of conservation of gross anatomy with humans and sophisticated gene manipulation techniques made possible by homologous recombination [47]. Although application of gene targeting by homologous recombination has been explored in zebrafish, these attempts have only met with little success, mainly due to the absence of proper embryonic stem cells [46]. However, other methods to manipulate gene function have gained prominence in the zebrafish. Morpholino antisense oligonucleotides and TILLING are two main reverse genetics methods, and now artificial endonuclease enzymes, ZFNs and TALENs, are emerging as a new technique to study specific genes of interest.

\section{Morpholino knockdown}

RNAi mediated gene knockdown technique is widely used in a variety of model systems from cell cultures to mammalian systems. Although there have been a few successful efforts to apply this technique in the zebrafish, RNAi mediated gene knockdown is not widely applicable in the zebrafish [24,35]. Instead, knockdown of gene function by morpholino antisense nucleotides is the most widely used reverse genetics technique to study genes of interest in the zebrafish [1]. Morpholinos are chemically synthesized nucleotides with morpholine rings, thereby resistant to breakdown by nucleases [34]. Usually morpholino 
sequences are designed to bind in the vicinity of the start codon to block initiation of translation or to splice acceptor sequences to cause abberantly spliced mRNAs.

Morpholinos are injected into freshly fertilized eggs and effectively block mRNA translation or splicing of target genes up to 5dpf, before becoming too diluted to efficiently interfere with translation. At this stage of development, most organs, including endocrines systems, are fully functional in zebrafish [29]. The biggest advantage of morpholinos is their ease of use and quick read-out. Together with the high fecundity of zebrafish, morpholinos can be injected into several hundreds of embryos in one experiment and resulting phenotypes are readily observed in those embryos a few days later (Figure 1). Furthermore, co-injection of a combination of up to three morpholinos can be performed to achieve double and triple knockdowns of genes of interest [31]. In addition, concentrations of morpholinos injected can be titrated in order to investigate dose-dependent resulting phenotypes. One exemplary application to the field of endocrinology is the downregulation of the zebrafish $\operatorname{irx} 3 a$ orthologue [39]. This transcriptional regulator is expressed in the kidney, hypothalamus and endoderm derived tissues. Morpholino-based downregulation of irx3a increased the mRNA expression level of ghrelin while decreased that of insulin, demonstrating an involvement of irx3a in the regulation of $\beta$ - and $\varepsilon$-cells of the pancreas.

Though highly efficient, knockdown experiments using morpholinos require optimization of the injection dose and careful observation of resulting phenotypes as there is a potential risk of off-target effects, which may cause unspecific phenotypes [10]. Also, the effectiveness of the downregulation needs to be carefully assessed, e.g. by demonstrating the reduction or absence of the target protein in western blots. Keeping these experimental precautions in mind, morpholino-based knockdown is one of the most advantageous techniques the zebrafish has to offer. In addition to the conventional knockdown of translation of target 
mRNAs, morpholinos can also be used to block the maturation of microRNAs (miRNAs) as well as to inhibit their binding to the target mRNAs, facilitating assessment of target genes of miRNAs [44].

\section{TILLING}

TILLING (targeted induced local lesions in genomes) is a combined method of forward and reverse genetics based on chemical mutagenesis to isolate mutants harboring point mutations in genes of interest [48]. Firstly, male fish are mutagenized by ENU (N-Nitroso-N-ethylurea), a chemical mutagen, and subsequently, mutations in target genes are sought by sequencing the target regions from genomes extracted from the mutagenized individuals (Figure 2A). Although TILLING is a technically efficient method to obtain fish with mutations in genes of interest, since it requires a large-scale sequencing of a large pool of individual fish, it is difficult to conduct as a routine technique in most individual laboratories. Therefore, community-based zebrafish mutation projects by TILLING were established by several consortia (eg. Sanger Institute) and the information and resources are shared via the zebrafish community database (http://zfin.org/).

\section{ZFNs}

Zinc finger nucleases (ZFNs) are artificial endonuclease enzymes originally introduced as a hybrid restriction enzyme [25]. ZFNs consist of two domains. One is the zinc finger motif domain that recognizes a specific sequence of genomic DNA and the other is the FokI restriction enzyme domain that cuts double-stranded DNA [11]. A DNA double-stranded break is repaired by homologous recombination or a non-homologous end joining (NHEJ). 
As NHEJ is an error-prone process, it creates small deletion/insertion ('indel') mutations in a site of lesion. Thus, by engineering the zinc finger domains to bind specific loci of genomes, it is possible to introduce mutations into genes of interest.

ZFNs are injected into fertilized eggs at the one to two cell stages. Individuals harboring mutations in a target gene are subsequently identified and these founder fish are outcrossed to obtain an F1 generation. After confirming the mutations in the F1 generation, they are incrossed and the resulting F2 generation with a homozygous mutation in a gene of interest can be subject to analysis of the gene function. Although genes in the endocrine systems of zebrafish have not yet been targeted by ZFNs, there is a growing list of zebrafish lines generated by this technology [27].

Targeted mutagenesis by ZFNs is simple in theory and ZFNs are indeed a viable tool for targeted mutagenesis, but the production of validated ZFN pairs is technically challenging and costly. In addition, ZFNs have a potential risk to cause off-target effects, which may yield unspecific phenotype unrelated to the function of target genes [36]. One of the disadvantages of ZFNs is their complexity regarding the designing of DNA binding domains [50]. ZFN binding domain contains 3 to 4 DNA recognition modules, each of which recognizes 3 DNA bases. However, when all the modules are assembled as one DNA binding domain, the specificity of DNA binding can decrease and therefore, designing specific ZFN pairs that recognize specific loci of the genome is selective, making some genes unavailable for targeted knockout via ZFN.

TALENs 
Based on the same principal as ZFNs, the transcription activator-like effector nucleases (TALENs) are emerging as a new and efficient tool for targeted mutagenesis [5]. TALENs use the same functional domains, namely the restriction enzyme domain and the DNA binding domain. In the case of TALENs, the DNA binding domain is derived from plant pathogenic bacteria with TALE modules recognizing specific bases (Figure 2B) [4]. TALENs are far more flexible and much simpler to generate than ZFNs because each TALE module of the DNA recognition domain of TALENs consists of a cipher for one particular DNA base and can be easily assembled one by one. Although off-target effects of TALENs still need to be evaluated with more accumulating experience, TALENs are increasingly used as an easily accessible gene targeting technique for many laboratories.

\section{Transgenic Approaches}

Tissue specific expression of a transgene in in vivo system is a powerful tool for genetic studies. Transgenesis was historically the weakness of the zebrafish model system, but owing to extensive efforts to improve transgenesis in zebrafish, it turned into one of the most appealing features of zebrafish. There are several ways to enhance transgenesis in zebrafish, such as co-injection of I-SceI meganuclease, but the most common method currently largely in use is utilization of the Tol2 transposon system [14,22]. The Tol2 transposable elements were originally discovered in the Japanese medaka Oryzias latipes [23]. Co-injection of expression constructs containing transgenes flanked by the Tol2 elements with the transposase mRNAs greatly enhances the efficiency of transgenesis in zebrafish, including germ line transmission (Figure 3A). Together with the development of the gateway technology that significantly facilitates flexible assemblies of expression constructs, researchers can now fully exploit advantages of the zebrafish as a model system [26]. 


\section{GAL4/UAS}

The GAL4/UAS system is a standard approach in Drosophila [9]. Since the improvement of the transgenesis in zebrafish by the Tol2 system, use of the GAL4/UAS system has been becoming a more and more common approach in zebrafish [40,41]. By fusing a transgene of interest under the control of the UAS promoter, the GAL4/UAS system allows tissue specific expression of the transgene when combined with the tissue specific GAL4 driver line (Figure 3B). Using this technology, overexpression of a gene of interest with tissue specificity can be achieved. The most advantageous feature of the GAL4/UAS system is that an array of GAL4 driver lines can be combined with a range of UAS effector lines, allowing a multitude of spatiotemporal regulation of gene functions. The stable UAS transgenic zebrafish lines generated so far express fluorophores in GAL4 positive cells to visualize gene function and cell specific behaviors. Future work is expected to produce various UAS effector lines that activate or silence gene function under an array of multiple GAL4 driver lines [33].

Heat shock promoters

Heat shock promoters drive expression of transgenes when the surrounding temperature is raised to $38^{\circ} \mathrm{C}$, allowing temporal and spatial control of transgene expression (Figure 3C) [17]. This technique is a powerful tool when studying effects of overexpression of genes of interest at different time points during the development. In addition, being a small waterliving animal, the zebrafish is a particularly viable model system for this technique as the surrounding temperature is easily controlled [42]. One issue entailing the use of the heat shock promoters is a leakiness of the promoter activity, resulting in the basal expression of the downstream genes even at the permissive temperature [19]. However, this leakiness can 
be circumvented by integrating this technique in the ligand-inducible Cre/loxP system [18]. Furthermore, a method using a modified soldering iron has been developed to enable a sitespecific heat induction in order to achieve spatial specificity of transgene control as well as temporal specificity $[20,38]$.

\section{Cre/loxP system}

One of the remaining challenges in zebrafish reverse genetics is to accomplish a conditional knockout/down technique (Figure 3D). In that respect, the mouse model is still the most sophisticated model system due to the well-established Cre/loxP system, by which mouse geneticists can manipulate genes of interest in a spatiotemporal manner at will [2]. It is a natural course of progress to apply the Cre/loxP system to the zebrafish model system to achieve the spatiotemporal control of gene expression, but shortage of a promoter/enhancer that ubiquitously drive expression of transgenes has been an obstacle [21,49]. Although several elements that are assumed to have broad control of genes have been identified, including $h 2 a f x$, tbp and $\beta$-actin promoter, these expression driving elements are inactivated during the development and thus cannot be a proper ubiquitous promoter [3].

Recently, the promoter of the zebrafish ubiquitin, a ubiquitin peptide precursor, that is highly conserved from plants to humans, was isolated and shown to drive expression of transgenes at all stages of the zebrafish development and in all adult organs [32]. Subsequently, stable EGFP transgenic lines were generated using the ubiquitin promoter that showed strong ubiquitous expression of EGFP from an early stage of development to adulthood.

Furthermore, the same group created $u b i: c r e^{E R t 2}$ transgenic lines and detected Cre activity at various time points of development upon administration of tamoxifen. By crossing this 
ubi:cre ${ }^{E R t 2}$ lines with $u b i$ reporter transgenics flanked by two loxP sites, a strong Cre mediated reporter activity was successfully demonstrated.

Although the $u b i$ promoter activity reported in this study varies depending on cell types in different tissues and loxP reporter activity upon Cre exposure has positional effects, isolation of a ubiquitous promoter and application of the Cre/loxP system in zebrafish has demonstrated the potential of this animal model to become a more sophisticated and flexible system in the near future.

Targeted cell ablation

Cell type specific ablation is an important tool for regeneration studies and enhanced transgenesis made targeted cell ablation one of the powerful methods in zebrafish. Bacterial nitroreductase (NTR) converts metronidazole (Met) into a cytotoxic agent [6]. By expressing NTR under a promoter that drives expression in a cell type specific manner, cell populations of interest can be ablated in a time point specific manner upon administration of Met. One example of the application of this technique is that insulin producing $\beta$-cells were successfully ablated in the pancreas, resulting in reduced mass of $\beta$-cells [37]. This technique can be combined with the GAL4/UAS system, where NTR is expressed under the UAS promoter, which is transactivated by the GAL4 driver in a particular cell type [15]. With this combination, targeted cell ablation can be performed with more flexibility.

\section{Conclusion}

The zebrafish offers a diverse range of approaches to study human diseases. Recent advancements in reverse genetics technologies further accelerate the use of the zebrafish as a 
model system. Many of the key factors in endocrine systems are conserved from humans to zebrafish and therefore, considering the useful biological features, together with reverse genetics techniques in hand, the zebrafish should attract more attention as a major organism to study endocrinology. Endocrinology research using zebrafish has the potential to provide more insights into our understanding of human endocrine systems and contribute to elucidations of genetic mechanisms of human diseases caused by disruptions of the endocrine systems.

\section{Acknowledgements}

We apologize in advance to all the researchers whose work we could not cite due to space limitations. Work in the authors' lab is supported by the Swiss National Science Foundation (31003A_135598), EU ZF-HEALTH $7^{\text {th }}$ frame work program and the Zurich Center for Integrative Human Physiology.

\section{References}

[1] V.M. Bedell, S.E. Westcot, S.C. Ekker, Lessons from morpholino-based screening in zebrafish, Brief Funct Genomics 10 (2011) 181-188.

[2] C.S. Branda, S.M. Dymecki, Talking about a revolution: The impact of site-specific recombinases on genetic analyses in mice, Dev Cell 6 (2004) 7-28.

[3] C.T. Burket, J.E. Montgomery, R. Thummel, S.C. Kassen, M.C. LaFave, D.M. Langenau, L.I. Zon, D.R. Hyde, Generation and characterization of transgenic zebrafish lines using different ubiquitous promoters, Transgenic Res 17 (2008) 265279.

[4] L. Cade, D. Reyon, W.Y. Hwang, S.Q. Tsai, S. Patel, C. Khayter, J.K. Joung, J.D. 
Sander, R.T. Peterson, J.R. Yeh, Highly efficient generation of heritable zebrafish gene mutations using homo- and heterodimeric TALENs, Nucleic Acids Res 40 (2012) 8001-8010.

[5] K.J. Clark, D.F. Voytas, S.C. Ekker, A TALE of two nucleases: gene targeting for the masses?, Zebrafish 8 (2011) 147-149.

[6] S. Curado, D.Y. Stainier, R.M. Anderson, Nitroreductase-mediated cell/tissue ablation in zebrafish: a spatially and temporally controlled ablation method with applications in developmental and regeneration studies, Nat Protoc 3 (2008) 948-954.

[7] Y. Doyon, J.M. McCammon, J.C. Miller, F. Faraji, C. Ngo, G.E. Katibah, R. Amora, T.D. Hocking, L. Zhang, E.J. Rebar, P.D. Gregory, F.D. Urnov, S.L. Amacher, Heritable targeted gene disruption in zebrafish using designed zinc-finger nucleases, Nat Biotechnol 26 (2008) 702-708.

[8] W. Driever, L. Solnica-Krezel, A.F. Schier, S.C. Neuhauss, J. Malicki, D.L. Stemple, D.Y. Stainier, F. Zwartkruis, S. Abdelilah, Z. Rangini, J. Belak, C. Boggs, A genetic screen for mutations affecting embryogenesis in zebrafish, Development 123 (1996) $37-46$.

[9] J.B. Duffy, GAL4 system in Drosophila: a fly geneticist's Swiss army knife, Genesis $34(2002) 1-15$.

[10] J.S. Eisen, J.C. Smith, Controlling morpholino experiments: don't stop making antisense, Development 135 (2008) 1735-1743.

[11] S.C. Ekker, Zinc finger-based knockout punches for zebrafish genes, Zebrafish 5 (2008) 121-123.

[12] M.C. Fishman, Genomics. Zebrafish--the canonical vertebrate, Science 294 (2001) $1290-1291$.

[13] J.E. Foley, M.L. Maeder, J. Pearlberg, J.K. Joung, R.T. Peterson, J.R. Yeh, Targeted 
mutagenesis in zebrafish using customized zinc-finger nucleases, Nat Protoc 4 (2009) $1855-1867$.

[14] C. Grabher, J. Wittbrodt, Recent advances in meganuclease-and transposon-mediated transgenesis of medaka and zebrafish, Methods Mol Biol 461 (2008) 521-539.

[15] C. Gray, C.A. Loynes, M.K. Whyte, D.C. Crossman, S.A. Renshaw, T.J. Chico, Simultaneous intravital imaging of macrophage and neutrophil behaviour during inflammation using a novel transgenic zebrafish, Thromb Haemost 105 (2011) 811819.

[16] P. Haffter, M. Granato, M. Brand, M.C. Mullins, M. Hammerschmidt, D.A. Kane, J. Odenthal, F.J. van Eeden, Y.J. Jiang, C.P. Heisenberg, R.N. Kelsh, M. Furutani-Seiki, E. Vogelsang, D. Beuchle, U. Schach, C. Fabian, C. Nusslein-Volhard, The identification of genes with unique and essential functions in the development of the zebrafish, Danio rerio, Development 123 (1996) 1-36.

[17] M.C. Halloran, M. Sato-Maeda, J.T. Warren, F. Su, Z. Lele, P.H. Krone, J.Y. Kuwada, W. Shoji, Laser-induced gene expression in specific cells of transgenic zebrafish, Development 127 (2000) 1953-1960.

[18] S. Hans, D. Freudenreich, M. Geffarth, J. Kaslin, A. Machate, M. Brand, Generation of a non-leaky heat shock-inducible Cre line for conditional Cre/lox strategies in zebrafish, Dev Dyn 240 (2011) 108-115.

[19] S. Hans, J. Kaslin, D. Freudenreich, M. Brand, Temporally-controlled site-specific recombination in zebrafish, PLoS One 4 (2009) e4640.

[20] M.E. Hardy, L.V. Ross, C.B. Chien, Focal gene misexpression in zebrafish embryos induced by local heat shock using a modified soldering iron, Dev Dyn 236 (2007) 3071-3076.

[21] S. Higashijima, H. Okamoto, N. Ueno, Y. Hotta, G. Eguchi, High-frequency 
generation of transgenic zebrafish which reliably express GFP in whole muscles or the whole body by using promoters of zebrafish origin, Dev Biol 192 (1997) 289-299.

[22] K. Kawakami, Transgenesis and gene trap methods in zebrafish by using the Tol2 transposable element, Methods Cell Biol 77 (2004) 201-222.

[23] K. Kawakami, A. Shima, N. Kawakami, Identification of a functional transposase of the Tol2 element, an Ac-like element from the Japanese medaka fish, and its transposition in the zebrafish germ lineage, Proc Natl Acad Sci U S A 97 (2000) 11403-11408.

[24] A. Kelly, A.F. Hurlstone, The use of RNAi technologies for gene knockdown in zebrafish, Brief Funct Genomics 10 (2011) 189-196.

[25] Y.G. Kim, J. Cha, S. Chandrasegaran, Hybrid restriction enzymes: zinc finger fusions to Fok I cleavage domain, Proc Natl Acad Sci U S A 93 (1996) 1156-1160.

[26] K.M. Kwan, E. Fujimoto, C. Grabher, B.D. Mangum, M.E. Hardy, D.S. Campbell, J.M. Parant, H.J. Yost, J.P. Kanki, C.B. Chien, The Tol2kit: a multisite gateway-based construction kit for Tol2 transposon transgenesis constructs, Dev Dyn 236 (2007) 3088-3099.

[27] N.D. Lawson, S.A. Wolfe, Forward and reverse genetic approaches for the analysis of vertebrate development in the zebrafish, Dev Cell 21 (2011) 48-64.

[28] G.J. Lieschke, P.D. Currie, Animal models of human disease: zebrafish swim into view, Nat Rev Genet 8 (2007) 353-367.

[29] H. Lohr, M. Hammerschmidt, Zebrafish in endocrine systems: recent advances and implications for human disease, Annu Rev Physiol 73 (2011) 183-211.

[30] I.M. McGonnell, R.C. Fowkes, Fishing for gene function--endocrine modelling in the zebrafish, J Endocrinol 189 (2006) 425-439.

[31] C.L. McNulty, J.N. Peres, N. Bardine, W.M. van den Akker, A.J. Durston, 
Knockdown of the complete Hox paralogous group 1 leads to dramatic hindbrain and neural crest defects, Development 132 (2005) 2861-2871.

[32] C. Mosimann, C.K. Kaufman, P. Li, E.K. Pugach, O.J. Tamplin, L.I. Zon, Ubiquitous transgene expression and Cre-based recombination driven by the ubiquitin promoter in zebrafish, Development 138 (2011) 169-177.

[33] A. Muto, K. Kawakami, Imaging functional neural circuits in zebrafish with a new GCaMP and the Gal4FF-UAS system, Commun Integr Biol 4 (2011) 566-568.

[34] A. Nasevicius, S.C. Ekker, Effective targeted gene 'knockdown' in zebrafish, Nat Genet 26 (2000) 216-220.

[35] A.C. Oates, A.E. Bruce, R.K. Ho, Too much interference: injection of doublestranded RNA has nonspecific effects in the zebrafish embryo, Dev Biol 224 (2000) 20-28.

[36] V. Pattanayak, C.L. Ramirez, J.K. Joung, D.R. Liu, Revealing off-target cleavage specificities of zinc-finger nucleases by in vitro selection, Nat Methods 8 (2011) 765770.

[37] H. Pisharath, J.M. Rhee, M.A. Swanson, S.D. Leach, M.J. Parsons, Targeted ablation of beta cells in the embryonic zebrafish pancreas using E. coli nitroreductase, Mech Dev 124 (2007) 218-229.

[38] M. Placinta, M.C. Shen, M. Achermann, R.O. Karlstrom, A laser pointer driven microheater for precise local heating and conditional gene regulation in vivo. Microheater driven gene regulation in zebrafish, BMC Dev Biol 9 (2009) 73

[39] A. Ragvin, E. Moro, D. Fredman, P. Navratilova, O. Drivenes, P.G. Engstrom, M.E. Alonso, E. de la Calle Mustienes, J.L. Gomez Skarmeta, M.J. Tavares, F. Casares, M. Manzanares, V. van Heyningen, A. Molven, P.R. Njolstad, F. Argenton, B. Lenhard, T.S. Becker, Long-range gene regulation links genomic type 2 diabetes and obesity 
risk regions to HHEX, SOX4, and IRX3, Proc Natl Acad Sci U S A 107 (2010) 775780.

[40] N. Scheer, J.A. Campos-Ortega, Use of the Gal4-UAS technique for targeted gene expression in the zebrafish, Mech Dev 80 (1999) 153-158.

[41] E.K. Scott, The Gal4/UAS toolbox in zebrafish: new approaches for defining behavioral circuits, J Neurochem 110 (2009) 441-456.

[42] W. Shoji, M. Sato-Maeda, Application of heat shock promoter in transgenic zebrafish, Dev Growth Differ 50 (2008) 401-406.

[43] I. Skromne, V.E. Prince, Current perspectives in zebrafish reverse genetics: moving forward, Dev Dyn 237 (2008) 861-882.

[44] A.A. Staton, A.J. Giraldez, Use of target protector morpholinos to analyze the physiological roles of specific miRNA-mRNA pairs in vivo, Nat Protoc 6 (2011) 2035-2049.

[45] G. Streisinger, C. Walker, N. Dower, D. Knauber, F. Singer, Production of clones of homozygous diploid zebra fish (Brachydanio rerio), Nature 291 (1981) 293-296.

[46] L. Sun, C.S. Bradford, C. Ghosh, P. Collodi, D.W. Barnes, ES-like cell cultures derived from early zebrafish embryos, Mol Mar Biol Biotechnol 4 (1995) 193-199.

[47] K.R. Thomas, M.R. Capecchi, Site-directed mutagenesis by gene targeting in mouse embryo-derived stem cells, Cell 51 (1987) 503-512.

[48] E. Wienholds, F. van Eeden, M. Kosters, J. Mudde, R.H. Plasterk, E. Cuppen, Efficient target-selected mutagenesis in zebrafish, Genome Res 13 (2003) 2700-2707.

[49] S. Yoshikawa, K. Kawakami, X.C. Zhao, G2R Cre reporter transgenic zebrafish, Dev Dyn 237 (2008) 2460-2465.

[50] C. Zhu, T. Smith, J. McNulty, A.L. Rayla, A. Lakshmanan, A.F. Siekmann, M. Buffardi, X. Meng, J. Shin, A. Padmanabhan, D. Cifuentes, A.J. Giraldez, A.T. Look, 
J.A. Epstein, N.D. Lawson, S.A. Wolfe, Evaluation and application of modularly assembled zinc-finger nucleases in zebrafish, Development 138 (2011) 4555-4564. 


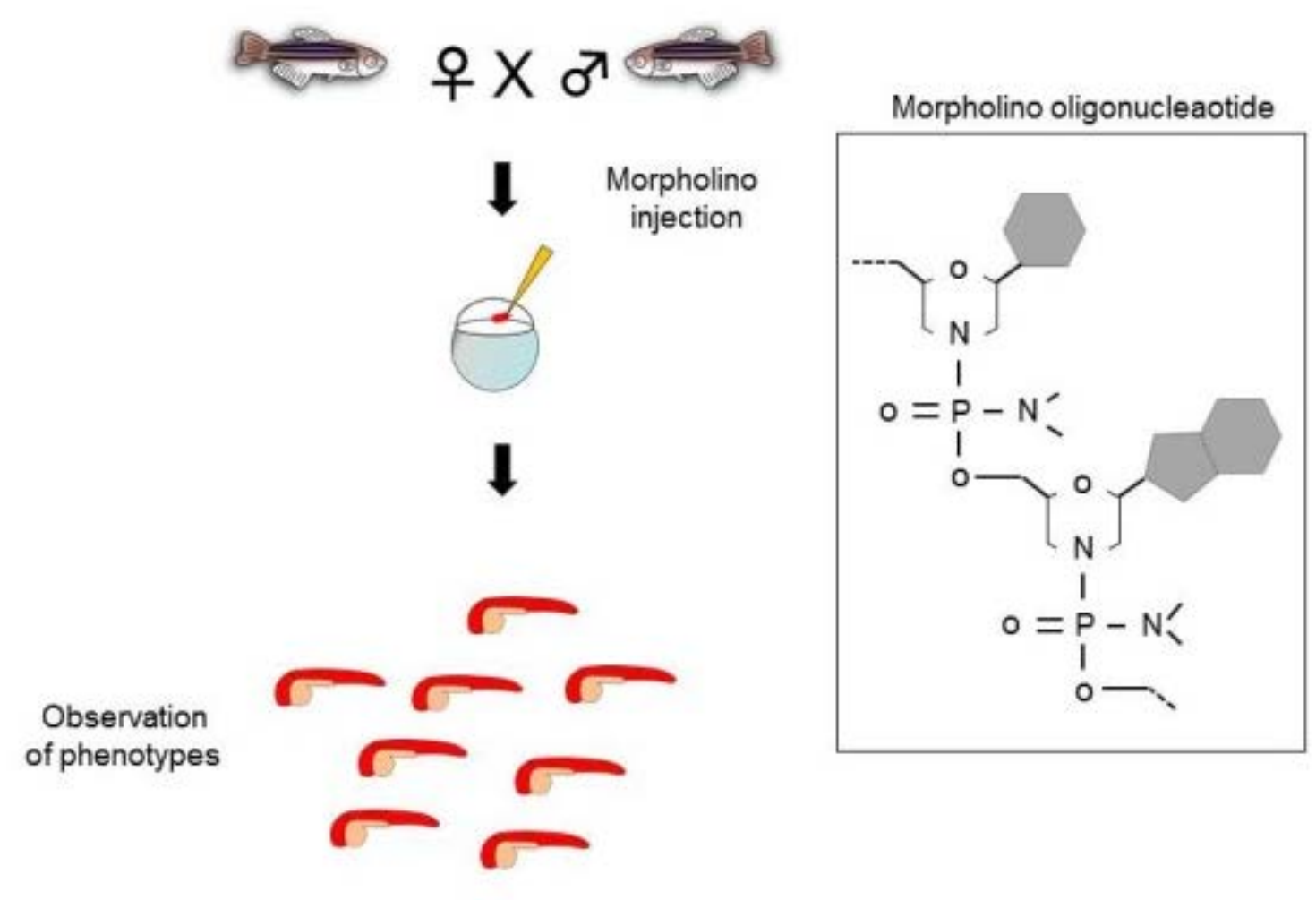

Figure 1. Overview of a knockdown experiment by morpholino antisense nucleotides. A chemical structure of a morpholino is shown. Morpholinos are normally injected into fertilized embryos at the one cell stage. Effects of knockdowns of target genes are readily observed in these embryos up to $5 \mathrm{dpf}$. 


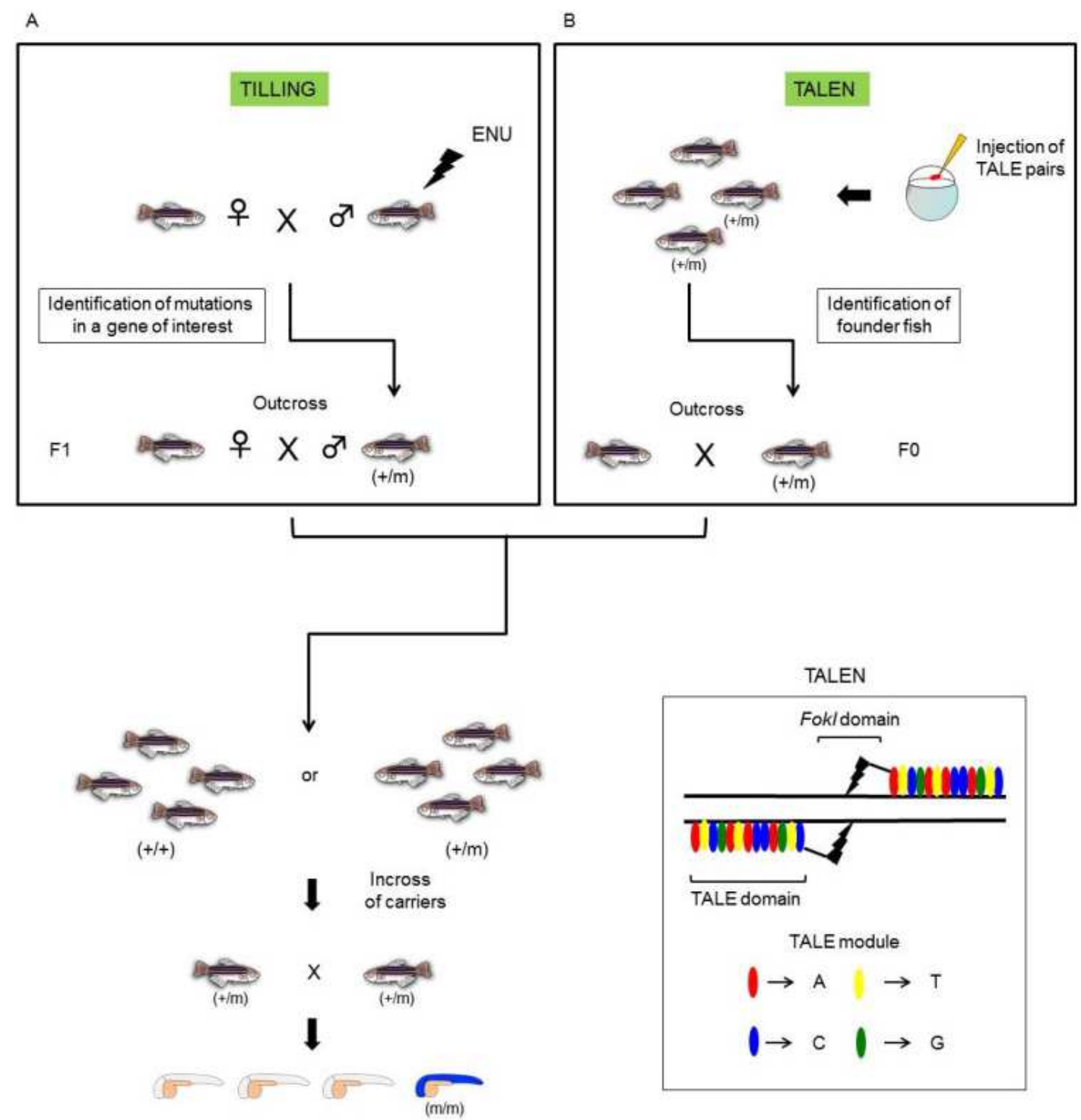

Figure 2. Schematic representation of procedures to obtain knockout zebrafish by TILLING and TALENs. (A) In a conventional TILLING approach, as a first step, male fish are mutagenized by ENU and outcrossed to obtain the F1 founder generation. The resulting F1 fish are screened (e.g. by PCR based techniques and sequencing) for individuals with mutations in genes of interest. The mutation carriers are then crossed with wild type female fish to obtain the F2 generation. Incrossing of the F2 families results in generation of the F3 fish with homozygous mutations in target genes. "m" indicates a mutation in genes of interest. (B) In a TALEN approach, gene specific TALENs are injected into fertilized embryos at the one to two cell stage. These embryos are raised to adulthood and individual fish carrying mutations (founder fish) are screened. The founder fish are then crossed with wild type fish to obtain the F1 generation. Incrossing of the F1 families results in the F2 families that include fish that are homozygous for mutations in target genes. Of note, it is one generation shorter to obtain homozygote fish in the TALEN approach than the TILLING approach. A schematic of a TALEN is also shown. Four colored ovals represent each TALE module of the DNA binding domain of TALENs that recognizes a specific base. 
A

Tol2 construct Co-injection of Tol2 constructs

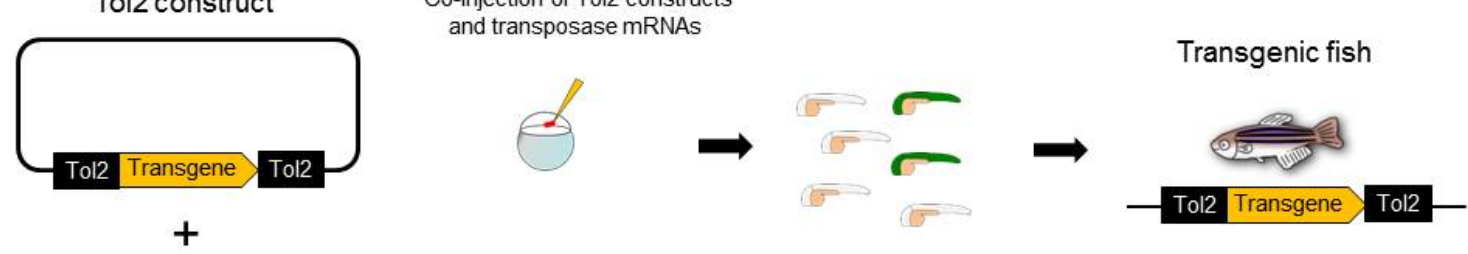

Transposase mRNA

B
GAL4 driver line
UAS reporter line

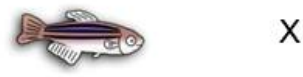

$x$
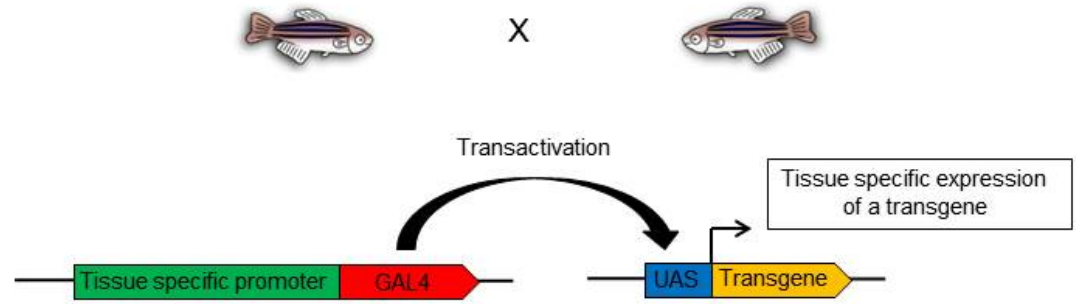

C
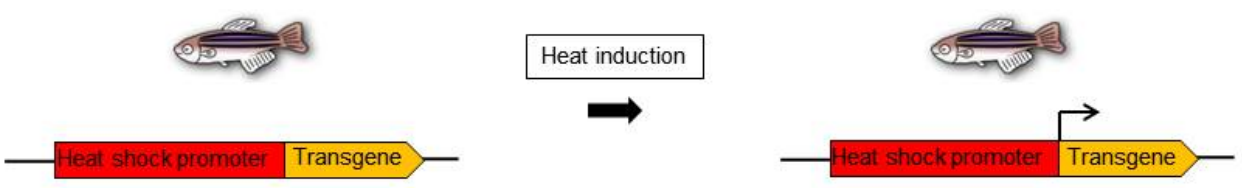

Temporal activation of a transgene

D

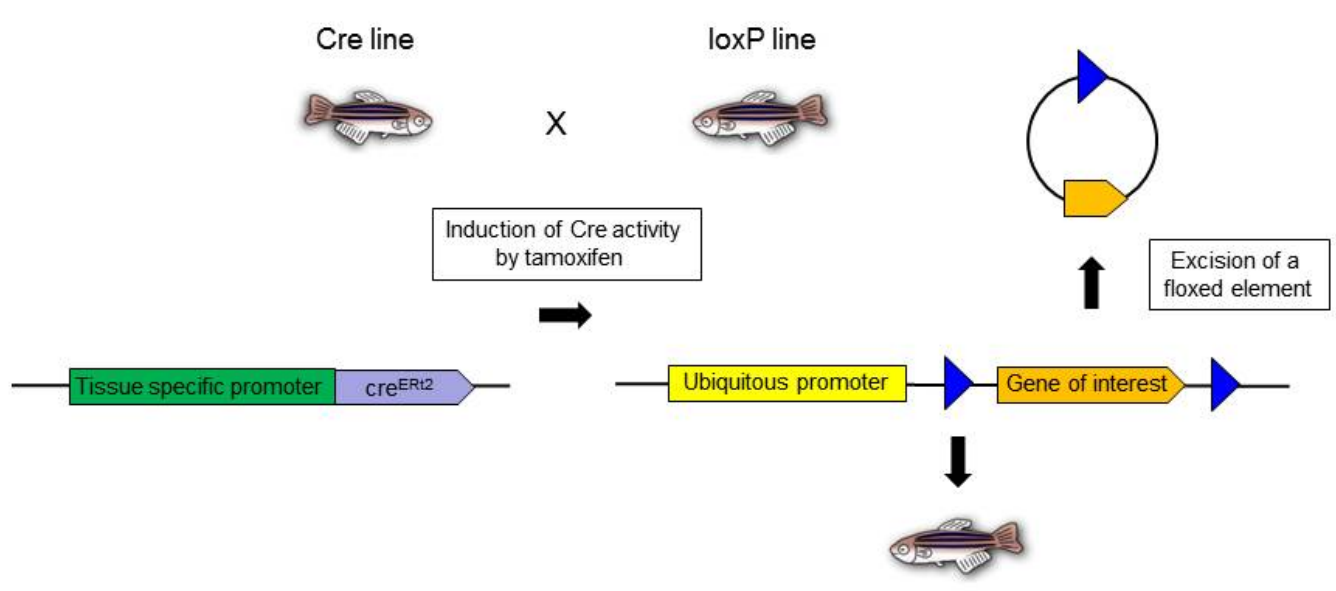

Tissue specific knockout fish 
Figure 3. Schemes for transgenic approaches. (A) Tol2 mediated transgenesis. Tol2 expression constructs contain transgenes flanked by the Tol 2 transposable elements. Coinjection of the Tol 2 constructs with mRNAs for transposase greatly enhances efficiency of integration of transgenes. (B) GAL4/UAS system. Upon crossing of a GAL4 driver line and UAS effector line, expression of GAL4 driven by a tissue specific promoter transactivates expression of a transgene through the UAS effector element in the resulting embryos. (C) Heat induced expression of a transgene. The heat shock promoter drives expression of a downstream transgene upon rise of the temperature. (D) Conditional knockout via the inducible Cre/loxP system. The Cre activity induced upon administration of tamoxifen excises a gene of interest flanked by loxP sites expressed under the ubiquitous promoter. By using a tissue specific promoter upstream of $c r e^{E R t 2}$, conditional tissue specific knockouts can be achieved. Blue triangles indicate the loxP element. 